\section{Overexpression of kynurenic acid and 3-hydroxyanthranilic acid after rat traumatic brain injury}

\author{
Arturo Mangas, ${ }^{1,2}$ Margarita Heredia, \\ Adelaida Riolobos, ${ }^{3}$ Antonio de la Fuente, ${ }^{3}$ \\ José María Criado, ${ }^{3}$ Javier Yajeya, ${ }^{3}$ \\ Michel Geffard, ${ }^{1}$ Rafael Coveñas ${ }^{2}$ \\ ${ }^{1}$ Gemacbio, Saint Jean d'Illac, France \\ ${ }^{2}$ Institute of Neurosciences of Castilla y \\ León (INCYL), Laboratory of \\ Neuroanatomy of the Peptidergic \\ Systems, University of Salamanca, Spain \\ ${ }^{3}$ School of Medicine, Department of \\ Physiology, University of Salamanca, \\ Spain
}

\section{Abstract}

Using an immunohistochemical technique, we have studied the distribution of kynuneric acid (KYNA) and 3-hydroxyanthranilic acid (3-HAA) in a rat brain injury model (trauma). The study was carried out inducing a cerebral ablation of the frontal motor cortex. Two mouse monoclonal specific antibodies previously developed by our group directed against KYNA and 3HAA were used. In control animals (shamoperated), the expression of both KYNA and 3-HAA was not observed. In animals in which the ablation was performed, the highest number of immunoreactive cells containing KYNA or 3-HAA was observed in the region surrounding the lesion and the number of these cells decreased moving away from the lesion. KYNA and 3-HAA were also observed in the white matter (ipsilateral side) located close to the injured region and in some cells placed in the white matter of the contralateral side. The distribution of KYNA and 3-HAA perfectly matched with the peripheral injured regions. The results found were identical independently of the perfusion date of animals (17, 30 or 54 days after brain injury). For the first time, the presence of KYNA and 3-HAA has been described in a rat trauma model. Moreover, by using a double immunocytochemistry protocol, it has been demonstrated that both metabolites were located in astrocytes. The findings observed suggest that, in cerebral trauma, KYNA and 3-HAA are involved in tissue damage and that these compounds could act, respectively, as a neuroprotector and a neurotoxic. This means that, in trauma, a counterbalance occurs and that a regulation of the indoleamine 2,3 dioxygenase (IDO) pathway could be required after a brain injury in order to decrease the deleterious effects of ending metabolites (the neurotoxic picolinic acid). Moreover, the localization of KYNA and 3-HAA in the contralateral side of the lesion suggests that the IDO pathway is also involved in the sprouting and pathfinding that follows a traumatic brain injury.

\section{Introduction}

Traumatic injuries are major health and socioeconomic problems affecting nowadays our societies..$^{1,2}$ Traumatic brain injury (trauma) is the leading cause of loss of human potential around the world, and especially in low and middle income countries. ${ }^{3}$ Severe lesions of motor cortex produce devastating effects, affecting voluntary movements. ${ }^{4}$ Almost one out of every ten deaths in the world were caused from injuries in 2010, higher than malaria, tuberculosis and immune deficiency syndrome combined together. ${ }^{5,6}$

Trauma occurring mechanisms are very similar to those occurring in neurodegenerative and ischemic processes. Ischemic stroke is characterized by glutamate excitotoxicity, excessive ROS production and inflammation, ${ }^{7-10}$ these processes also occur in traumatic brain injury. ${ }^{11}$ In stroke, indoleamine 2,3-dioxygenase (IDO) and nitric oxide (NO) pathways are activated and several intermediate metabolites of the IDO pathway such as kynurenic acid (KYNA) and 3-hydroxyanthranilic acid (3HAA) are overexpressed during ischemic stroke. ${ }^{8-10}$ KYNA, a tryptophan catabolite, is mainly synthesized by astrocytes, ${ }^{12,13}$ acts as a scavenger in these cells ${ }^{14}$ and, in ischemia, exerts a neuroprotective role against the neuronal loss. ${ }^{15}$ By contrast, 3HAA (another tryptophan catabolite) exerts a cytotoxic effect. ${ }^{16-18}$ In fact, 3-HAA inhibits mitochondria respiration, promotes oxidative damage on proteins and induces apoptosis. ${ }^{10,18-22}$

To our knowledge, the neuroanatomical distribution of the immunoreactive structures containing KYNA or 3-HAA in the mammalian central nervous system has been studied in an ischemic stroke model only and, in this model, the involvement of the IDO pathway has been demonstrated. ${ }^{8-10}$ Since mechanisms occurring in trauma are very similar to those appearing in stroke, ${ }^{23-}$ 24 our main aim is to study in a trauma model (ablation of the motor cortex) the presence / overexpression of markers (KYNA, 3-HAA) of the IDO pathway. This pathway has been implicated in some pathological mechanisms (e.g., inflammation). ${ }^{25-28}$ Thus, the presence, absence or overexpression of these molecules belong-
Correspondence: Dr. Arturo Mangas, University of Salamanca, Institute of Neurosciences of Castilla y León (INCYL), Laboratory of Neuroanatomy of the Peptidergic Systems (Lab. 14), c/Pintor Fernando Gallego 1, 37007 Salamanca, Spain. Tel. +34.923.294500 ext. 5315 -

Fax: +34.923.294549.

E-mail: mangasam@usal.es

Keywords: 3-OH-anthranilic acid; astrocyte; glia; immunohistochemistry; kynurenic acid; neurotoxicity; trauma.

Acknowledgments: This work has been supported by Gemacbio S.A. Laboratories (Saint Jean d'Illac, France), IDRPHT (Talence, France) and by the "Programa XI: Financiación de Unidades de Excelencia de la Universidad de Salamanca" (Salamanca, Spain). The authors wish to thank Javier Blanco for technical assistance.

Received for publication: 3 October 2018. Accepted for publication: 2 November 2018.

This work is licensed under a Creative Commons Attribution-NonCommercial 4.0 International License (CC BY-NC 4.0).

(C) Copyright A. Mangas et al., 2018

Licensee PAGEPress, Italy

European Journal of Histochemistry 2018; 62:2985 doi:10.4081/ejh.2018.2985

ing to the IDO pathway will serve to increase our knowledge on the underlying mechanisms occurring during traumatic processes (that is, the involvement of the IDO pathway in brain trauma) and to suggest possible therapeutic strategies for the treatment of brain trauma.

\section{Materials and Methods}

\section{Animals and experimental groups}

Twenty-four adult male Wistar rats (Charles River, 200-220 g at arrival) were used. Animals were housed under standardized conditions of light and temperature for 7 days before the experiments started. Animals were fed ad libitum, except when the paw-reaching-for-food task was carried out. For this procedure, animals were maintained at $86-88 \%$ of their initial ad libitum weight. $^{4,29}$ The experimental procedure of this work was performed under the guidelines of the legal and ethics recommendations of French, Spanish and European laws (2010/63/EU). Moreover, this study was approved by the research commission of the University of Salamanca (Spain).

Animals were divided into two groups: operated (surgical procedure with ablation 
of the cortex, $\mathrm{n}=12$ ) and "sham-operated" animals (surgical procedure without ablation of the cortex, $n=12$ ). Animals of both groups were perfused at 17, 30 and 54 days after the surgical intervention.

\section{Ablation of frontal motor cortex}

The ablation of deeply anesthetized animals was conducted by aspiration of the motor cortex. Thus, ablation of the contralateral cortex to the preferred paw was conducted by using a stereotaxic apparatus taking as reference the Bregma suture in order to conduct the same lesion to all animals (Figure 1). Sham-operated animals were anesthetized, according to their weights, and operated like the animals of the operated group (crania, meninges), but no ablation of the cortex was conducted. As previously described, lesion effectiveness was verified 7 days after the operation. ${ }^{29}$

\section{Immunocytochemical study}

Once the model was carried out and the animals perfused $(17,30$ or 54 days), an immunocytochemical study was performed. As previously reported, ${ }^{8-10,29}$ animals were deeply anaesthetized with Equithesin and perfused with paraformaldehyde (PAF) (4\%); brains were dissected out after decapitation, post-fixed in $4 \%$ of PAF for $16 \mathrm{~h}$ and cryoprotected in sucrose. Using a freezing microtome, 40-45 $\mu \mathrm{m}$-thick brain coronal sections were obtained and processed for immunocytochemistry. ${ }^{8-10}$ As previously described $^{8-10,30,31}$ and in order to inactivate the endogenous peroxidases, sections were pre-incubated in a mixture solution of methanol $/ \mathrm{H}_{2} \mathrm{O}_{2}(2 \mathrm{v} / 1 \mathrm{v})$ for $30 \mathrm{~min}$. Then, sections were washed three times with PBS for $30 \mathrm{~min}$ and pre-incubated in a PBS solution containing $0.3 \%$ Triton $\mathrm{X}-100$ and supplemented with $1 \%$ of normal horse serum (mixture solution) for $30 \mathrm{~min}$. After that, sections were incubated for $1 \mathrm{~h}$ and $45 \mathrm{~min}$ at room temperature and overnight at $4^{\circ} \mathrm{C}$ in the mixture solution containing the primary antibodies: monoclonal anti-3-HAA or antiKYNA antibodies (diluted 1/1,000; Gemacbio, Saint Jean d'Illac, France), polyclonal rabbit anti-glial fibrillary acidic protein (GFAP) antibody (1/100; Dako, Glostrup, Denmark) or polyclonal goat antiionized calcium-binding adapter molecule 1 (IBA-1) antibody (1/1,500, Abcam, Cambridge, UK). Afterward, three washes were conducted in PBS for $30 \mathrm{~min}$ at room temperature. Then, sections were incubated with the corresponding biotinylated antimouse/goat/rabbit immunogammaglobulin (BA-1,000; BA-5,000; BA-9,200, Vector Labs, Burlingame, CA, USA) (1/200 in mixture solution) for $1 \mathrm{~h}$ at room temperature. Later, sections were washed three times with PBS for $30 \mathrm{~min}$ and incubated with the avidin-biotin-peroxidase complex (ABC, Vectastain PK-6,100) (1/100) for $1 \mathrm{~h}$ at room temperature. Then, sections were rinsed three times with PBS for $30 \mathrm{~min}$, and a later wash of at least $10 \mathrm{~min}$ in Tris-HCl buffer. Finally, during $10 \mathrm{~min}$ in darkness and room temperature, the peroxidase was developed with $\mathrm{H}_{2} \mathrm{O}_{2}$, and 3, 3 ' diaminobenzidine (chromogen).

The immunological characteristics of the primary antibodies (anti-KYNA, anti-3HAA) used here have been published previously. ${ }^{8-10}$ In order to confirm the specificity of the immunoreactivity observed here, the following histological controls were also conducted: i) omission of the primary and/or secondary antibodies, and ii) preabsorption of the anti-KYNA or anti-3HAA antibodies with an excess (100 $\mu \mathrm{g} / \mathrm{mL}$ ) of their corresponding antigens (KYNA or 3-HAA). In both cases no residual immunoreactivity was found. The specificity of the anti-IBA-1 antibody has been previously demonstrated ${ }^{9}$ and, here, the immunoreactivity also disappeared after its preabsorption with the corresponding antigen. Moreover, in order to demonstrate that the immunoreactivity found for KYNA and 3-HAA was located in astrocytes, a doublelabelling was conducted in sections previously developed with DAB (brown precipitate) for KYNA or 3-HAA. We followed a previous published protocol. ${ }^{8-10}$ Thus, 4chloro-1-naphol was used to show the presence of GFAP (blue precipitate) in those cells containing KYNA or 3-HAA and the immunocytochemistry procedure showed that double-labelling cells (GFAP-KYNA or GFAP-3-HAA) were astrocytes.

Finally, the stereotaxic atlas of Paxinos and Watson ${ }^{32}$ was used for nomenclature and mapping. Photomicrographs were obtained by using a Kyowa Unilux-12 microscope coupled to an Olympus DP50 digital camera. Only the brightness and contrast of the images were adjusted in order to improve the visualization of results with Adobe Photoshop Elements software.

\section{Results}

\section{Timeline signal}

In all animals of the operated group (surgical procedure with ablation of the motor cortex), the results found were identical, independently of the date in which rats were perfused $(17,30$ or 54 days after the ablation). In all animals of the sham-operated group (surgical procedure without ablation of the cortex), the results observed were also identical, independently of the perfusion date. However, these results were very different when comparing sham-operated and operated animals (Table 1). In both groups, four markers have been studied: KYNA and 3-HAA (to demonstrate the involvement of the IDO pathway), IBA-1(to

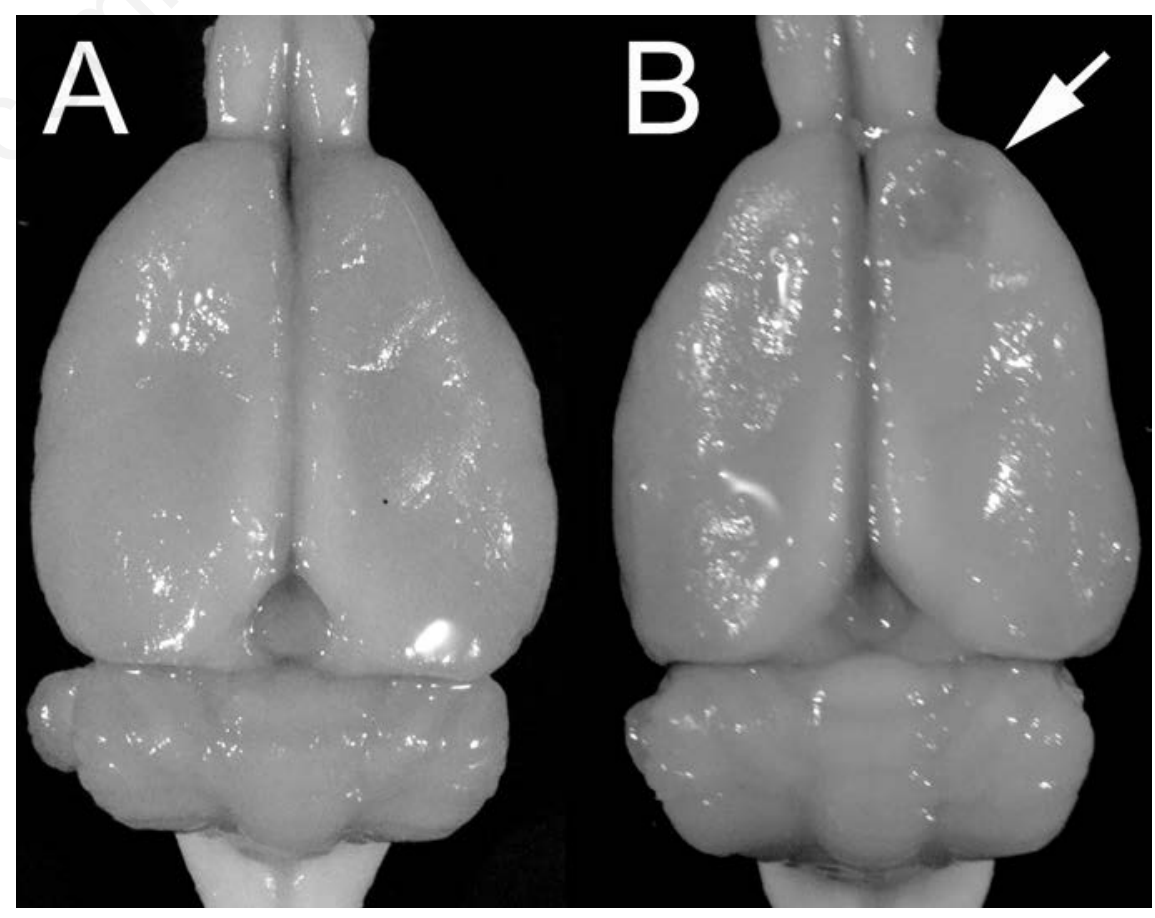

Figure 1. Low-power magnifications of brains after fixation. A) Sham operated animal; B) operated animal; the arrow indicates the lesion conducted on the motor cortex. 
check microglia activation) and GFAP (to confirm the presence of KYNA and 3-HAA in astrocytes).

\section{Sham-operated animals}

No immunolabelling was observed for KYNA and 3-HAA (Table 1), whereas a normal distribution of microglia (immunoreactivity for IBA-1) and astrocytes (immunoreactivity for GFAP) in both ipsilateral and contralateral sides was visualized (Table 1). Thus, after the surgical procedure without ablation of the motor cortex, the expression of KYNA and 3-HAA was not observed.

\section{Operated animals}

In the ipsilateral side, KYNA and 3HAA were exclusively observed in the region around the ablated area and in the motor cortex white matter placed close to the injured region. A high immunoreactivity (overexpression) for KYNA (Figure 2 and Figure 3 A,B) and 3-HAA (Figure 3 C,D and Figure 4) was found around this region, but the number of immunoreactive cells decreased moving away from the injured region (in fact, immunoreactivity disappeared in the regions of the white matter located far from the lesion) (Figure 2A and Figure 4A). Thus, a higher number of immunoreactive cells (containing KYNA or 3-HAA) was observed close to the lesion. In the white matter of the contralateral side, some cells containing KYNA or 3-HAA were also observed (Table 1). In comparison with sham-operated animals, an overexpression of the four markers studied (there is a perfect match between them) was observed in the region around the ablation (ipsilateral side) (Table 1) (Figures 2, 4, 5). As KYNA and 3-HAA, the expression of IBA-1 and GFAP decreased moving away from the region surrounding the lesion. In the white matter of the motor cortex placed far from the injured region, the expression of IBA-1 or GFAP was similar (normal distribution) to that found in the contralateral side of the operated animals and in sham-operated animals (Table 1).

\section{Astrocytes: Coexistence of GFAP with KYNA or 3-HAA}

Cells containing GFAP, KYNA or 3HAA showed the same morphological characteristics (Figures 2-4). In order to confirm the presence of KYNA and 3-HAA in astrocytes (GFAP), a double-immunolabelling technique was applied and GFAPimmunoreactivity was detected in astrocytes expressing KYNA or 3-HAA (Figure $3)$. The coexistence was observed in both ipsilateral and contralateral sides (operated animals).

\section{Discussion}

Here, it has been demonstrated for the first time that KYNA and 3-HAA were exclusively found in astrocytes of animals in which the ablation of the motor cortex was performed and that both molecules were overexpressed around the injured region. Moreover, the results observed (distribution/degree of the immunoreactivity) were identical in animals perfused 17, 30 or 54 days after the ablation.

\section{Anti-KYNA and anti-3-HAA labelling}

As previously described, ${ }^{8,10}$ monoclonal anti-KYNA and 3-HAA antibodies have been fully characterized by ELISA. Both antibodies were considered of high affinity $\left(10^{-10} \mathrm{M}\right.$ for KYNA; $10^{-9} \mathrm{M}$ for 3-HAA) and highly specifics, since they do not crossreact with close chemical structures. ${ }^{8,10}$ After the preabsorption of these antibodies with their corresponding antigens (antiKYNA with KYNA, anti-3-HAA with 3HAA) the immunoreactivity disappeared completely demonstrating that the immunolabelling observed was specific of the targeted molecules. Moreover, here, a double immunolabelling technique has been applied to demonstrate in astrocytes the coexistence of GFAP and KYNA or 3HAA. ${ }^{8-10,33}$ 4-chloro-1-napthol was used to

Table 1. Immunolabelling for kynurenic acid, 3-hydroxyanthranilic, ionized calciumbinding adapter molecule 1 and glial fibrillary acidic protein.

\begin{tabular}{lcccc} 
Marker & \multicolumn{2}{c}{ Sham-0perated } & \multicolumn{2}{c}{ Operated } \\
& Ipsilateral & Contralateral & Ipsilateral & Contralateral \\
Kynurenic acid & - & - & +++ & $+^{*}$ \\
3-hydroxyanthranilic & - & - & +++ & $+^{*}$ \\
$\begin{array}{l}\text { Ionized calcium-binding } \\
\text { adapter molecule 1 }\end{array}$ & + & + & +++ & + \\
\begin{tabular}{l} 
Glial fibrillary acidic protein \\
\hline
\end{tabular} & + & + & +++ & + \\
\hline
\end{tabular}

-, absence of immunoreactivity; + , presence; +*, only observed in some astrocytes located in the white matter of the motor cortex; +++ , very high: region around the ablation.
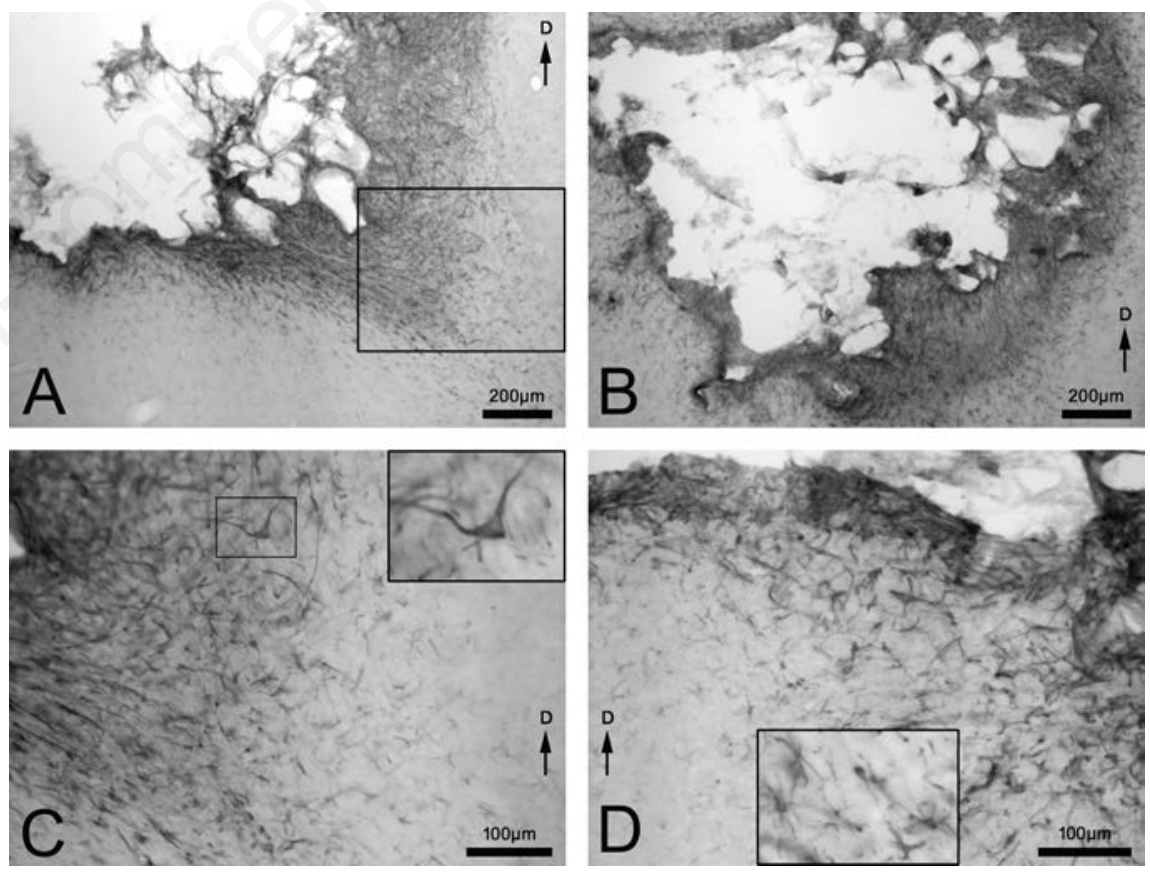

Figure 2. Immunoreactivity for KYNA. Ipsilateral side of the ablated motor cortex (A-D). A) Note the expression of KYNA in the region close to the lesion; the number of immunoreactive cells decreased moving away from the injured region. The region delimited by a rectangle is shown, at a higher magnification, in panel C. B) Picture of another region showing the immunoreactivity all around the lesion; $C$ ) The rectangle in the upper right corner is a higher magnification of the small left rectangle. D) High power magnification image, note the morphology of the cells containing KYNA. Small arrow D, dorsal orientation. 
show GFAP immunoreactivity in those sections in which the presence of KYNA or 3HAA had been observed by using diaminobenzidine. It is known that the diaminobenzidine reaction masks the antigen and catalytic sites of the first sequence of the immunoreagents, preventing an interaction of the second sequence with the reagents. ${ }^{8-10,33}$ Thus, in a neurotrauma model and thanks to the use of specific antibodies, it has been visualized for the first time the presence of KYNA and 3-HAA in rat astrocytes. This means that the IDO pathway is involved in the mechanisms occurring in brain trauma.

\section{Trauma versus transient middle cerebral artery occlusion}

Expression of KYNA and 3-HAA (peripheral area of the injured region and in the white matter of the ipsilateral and contralateral sides) occurred from at least 17 days after the ablation procedure. From early phases of stroke, it has been also described the expression of both molecules. ${ }^{8,10}$ Thus, in the transient middle cerebral artery occlusion model (tMCAO, stroke model), the expression of KYNA and 3-HAA was exclusively found around the infarcted region. ${ }^{8,10}$ However, in the trauma model, KYNA and 3-HAA were found around the injured region (there was a perfect anatomical match between both molecules) but also in astrocytes placed in the white matter of the ipsilateral and contralateral hemispheres. The immunolabelling found in the contralateral side was visualized only in a few cells. The presence of astrocytes (containing KYNA or 3-HAA) in the white matter could be due to a possible involvement of both molecules in mechanisms of neurogenesis and new axonal connections occurring after cortical ablation. ${ }^{29}$ The presence of KYNA and 3-HAA in the contralateral hemisphere supports the involvement of these molecules in the compensation mechanisms developed after a motor cortex injury. In a stroke model, it has been reported that sprouting neurons have many genes activated which are linked to axonal pathfinding or sprouting. ${ }^{34}$ This pathfinding processes are in close association with astrocytes and surrounding milieu. ${ }^{35-37}$ Thus, the role of the IDO pathway molecules (KYNA, 3-HAA) in the previous mentioned processes should be studied in-depth since it seems that this pathway plays an important role in the pathological processes involved in brain injury and repair.

In the neurotrauma model, the overexpression of KYNA, 3-HAA, IBA-1 and GFAP was observed around of the ablated cortex; this is in agreement with the results found in the tMCAO model in which a per-
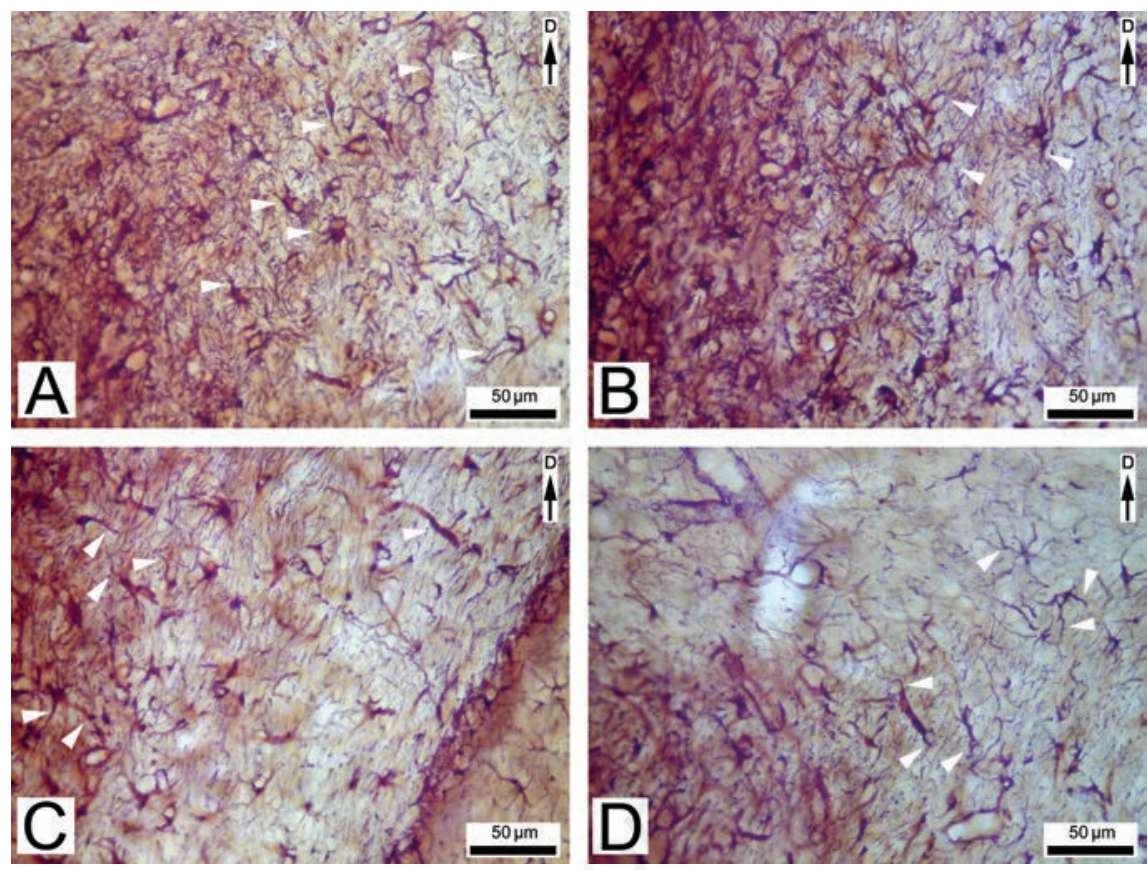

Figure 3. Photographs taken from the region around the lesion. Double-labelling of astrocytes containing GFAP and KYNA (A, B) or GFAP and 3-HAA (C, D). The immunolabelling for GFAP appears in blue (chloronapthol) and that of KYNA or 3-HAA in brown (diaminobenzidine). Note the blue staining in the astrocyte projections (arrowheads) and the brown staining in the cell bodies. Small arrow $\mathrm{D}$, dorsal orientation.
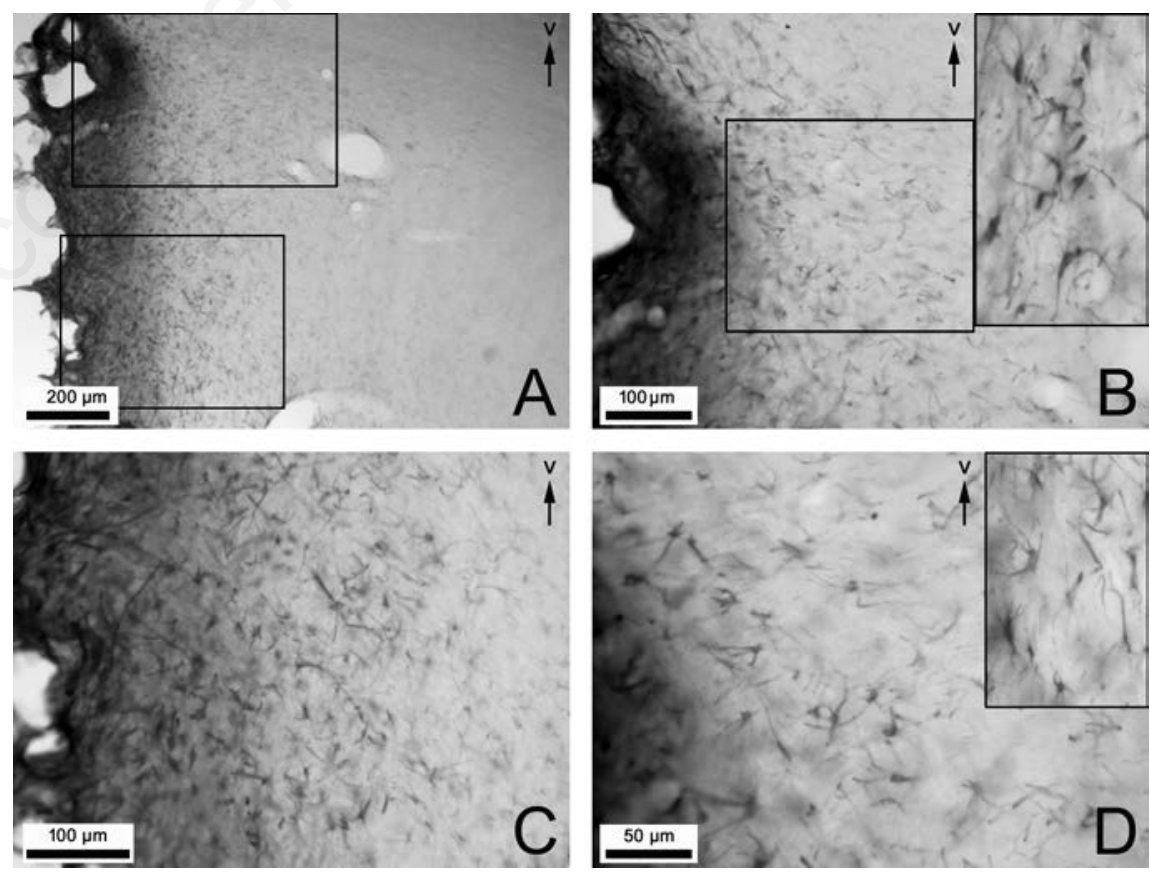

Figure 4. Immunoreactivity for 3-HAA. Ipsilateral side of the motor cortex. A) Note the expression of 3-HAA in the region close to the lesion; the number of immunoreactive cells decreased moving away from the injured region; the region delimited by the upper rectangle is shown at higher magnification in panel $\mathrm{B}$, and that of the lower rectangle in panel C. B) High-power magnification of the region delimited by the left rectangle is shown in panel $D$; right rectangle: a detail of another region showing immunoreactive cells. D) Rectangle, detail of cells containing 3-HAA. Small arrow V, ventral orientation. 
fect match of the four markers was also found in the ischemic area. Moreover, as previously described in the tMCAO model, ${ }^{8-10}$ here in astrocytes, it has been also demonstrated the coexistence GFAP and KYNA or 3-HAA.

In the tMCAO model, a great variability in the extension of the infarcted region has been reported, ${ }^{9,38}$ but in the neurotrauma model this great variability does not occur because the extension of the injured region is almost always the same. This does not occur because of the stereotaxic apparatus used when conducting the ablation; thus, the variability in the extension of the mechanical lesion is very limited in comparison to that observed in stroke.

\section{2,3 indoleamine dioxygenase pathway}

Both IDO pathway markers (KYNA and 3-HAA) have been found from 17 days after the surgical procedure to late phases (54 days after ablation). This pathway plays an important role in the immune response $\mathrm{e}^{39-41}$ and alterations of this pathway in several diseases (stroke, multiple sclerosis, Parkinson, Alzheimer, schizophrenia) have been reported. ${ }^{18,21,42,43}$

In the IDO pathway, KYNA and 3-HAA are products of the catabolism of tryptophan and it is known that they exert opposite actions: KYNA is a neuroprotector and 3HAA (a free radical generator) a cytotoxic. ${ }^{9,21,43-48}$ In the neurotrauma model used here, the expression of catabolites (KYNA, 3-HAA) of the IDO pathway is in agreement with the data reported in the tMCAO model. ${ }^{8-10}$ In the latter model, it is known that the administration of a new drug candidate (GEMST) reverted the expression of both KYNA and 3-HAA. ${ }^{9}$ Thus, based on the opposed exerted effects of both molecules, a mechanism directed to counterbalance the cytotoxic effect of the 3-HAA by an expression of KYNA could occur in both models (stroke, brain trauma). Moreover, KYNA acts as a glutamate receptor antagonist ${ }^{9}$ and hence the overexpression of KYNA in the neurotrauma model could block the deleterious actions exerted by glutamate. Our data also suggest that, after a brain injury, the enzymes kynureninase and microsomal hydroxylase (involved in the synthesis of 3-HAA) and kynurenine aminotransferases (involved in the synthesis of KYNA) are up-regulated in astrocytes. In the future, the inhibitors/activators of these enzymes might be investigated. It is also important to note that 3-HAA is an intermediary metabolite of the quinolinic acid, which is much more cytotoxic than 3HAA. ${ }^{47,49}$ Quinolinic acid induces the damage/death of glia cells and neurons and it is known that inhibitors of the enzyme 3-HAA oxidase (involved in the synthesis of quino- linic acid from 3-HAA) decreased the tissue damage promoted by the quinolinic acid. ${ }^{10}$ Therefore, after the ablation of the cortex an augmentation of the level of quinolinic acid could occur as it has been previously reported after spinal cord injury. ${ }^{50}$ Because of the intrinsic toxicity of quinolinic acid, in the future this point must be studied in-depth.

Our findings are in agreement with previous works. ${ }^{51-53}$ In the present study and in the tMCAO model, ${ }^{8,9}$ we have suggested that KYNA acts as a neuroprotector. In rodents, it has been reported that KYNA and kynurenate (a salt or ester of KYNA and an excitatory amino acid receptor antagonist) also exerted beneficial effects. ${ }^{51,53}$ In this sense, it is known that after a traumatic brain injury, the kynurenate decreased edema formation, improved motor deficits and attenuated the traumainduced cognitive dysfunction ${ }^{51}$ and that, before performing a traumatic brain injury, the in situ administration of KYNA attenuated rapid astroglial and microglial responses..$^{53}$ It is known that, after a traumatic brain injury, a rapid response of both astrocytes and microglia occurred. ${ }^{53}$ Moreover, the results found here, in a rat traumatic brain injury model, are in agreement with those observed in humans, since after a traumatic brain injury the levels of both KYNA and quinolinic acid increased in the human cere-
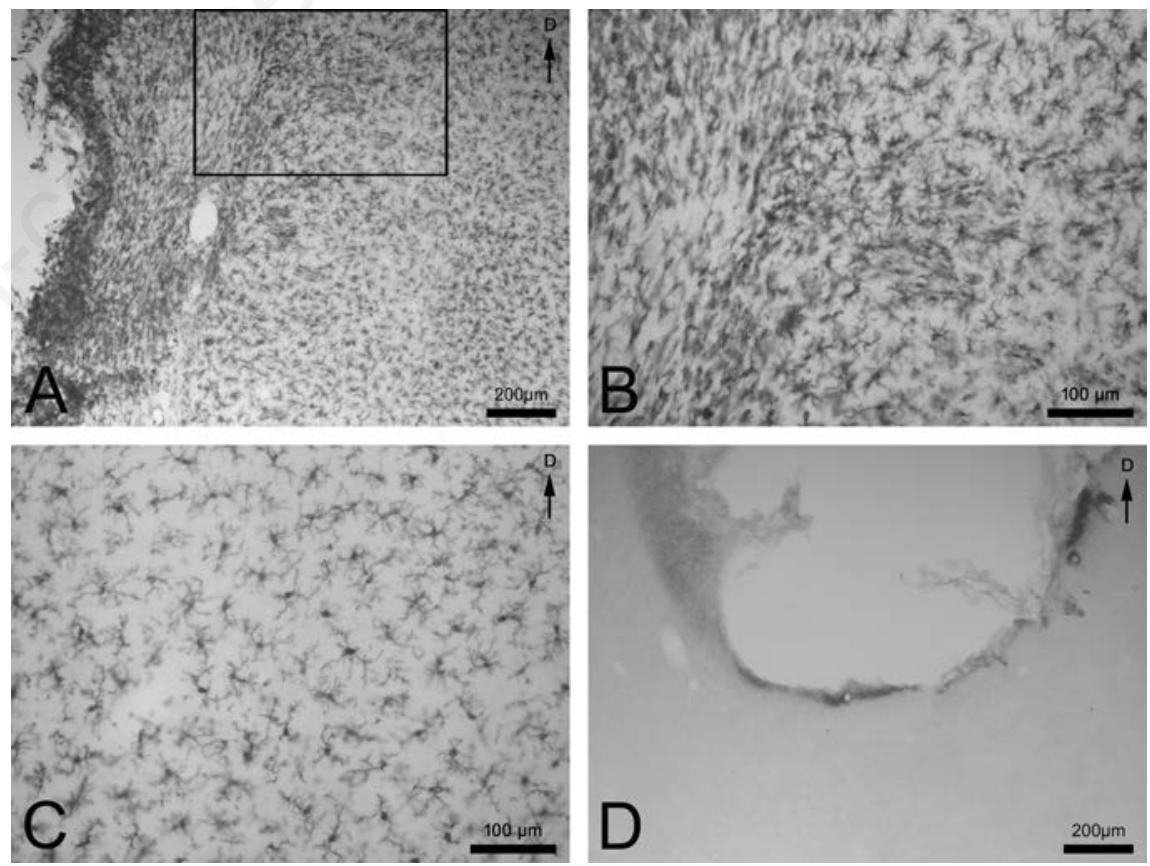

Figure 5. Immunoreactivity for IBA-1. A) High density of immunoreactive cells around the injured region. B) High-power magnification of the region delimited by the rectangle in A. C) Representative image of the labeling with IBA-1 observed in the contralateral side of the motor cortex or in sham-operated animals; compare the immunolabelling appearing in A and B. D) Control: absence of labelling when the first antibody was omitted in the immunocytochemical technique. Small arrow D, dorsal orientation. 
has been demonstrated the presence of astrocytes containing KYNA or 3-HAA; ii) KYNA and 3-HAA were exclusively found (overexpression) in the region around the ablation and in the white matter of both ipsilateral and contralateral sides; iii) there is a perfect match between KYNA and 3-HAA; the region around the ablation is also characterized by the overexpression of GFAP and IBA-1; iv) KYNA and 3-HAA are expressed from mild phases (17 days after the surgical procedure) to 54 days after ablation; v) the neuroprotective metabolite KYNA could be overexpressed in order to counterbalance the cytotoxic effect of 3HAA and its product (quinolinic acid), and vi) the presence of astrocytes containing KYNA and 3-HAA in the contralateral hemisphere means that they could be involved in the pathfinding and/or compensation mechanisms occurring after the ablation of the motor cortex. Finally, a suitable future experimentation could be the treatment of ablated animals with GEMST, a drug candidate for the treatment of stroke, since GEMST reverted the neuroanatomical changes (diminished the overexpression of KYNA, 3-HAA, IBA-1 and GFAP and exerted a general beneficial action) induced by an ischemic insult.

\section{References}

1. Maas AI, Stocchetti N, Bullock R. Moderate and severe traumatic brain injury in adults. Lancet Neurol 2008; 7:728-41.

2. Majdan M, Plancikova D, Brazinova A, Rusnak M, Nieboer D, Feigin V, et al. Epidemiology of traumatic brain injuries in Europe: a cross-sectional analysis. Lancet Public Health 2016;1 e76-e83.

3. Rubiano AM, Carney N, Chesnut R, Puyana JC. Global neurotrauma research challenges and opportunities. Nature 2015;527:S193-7.

4. Heredia M, Fuente A, Criado J, Yajeya J, Devesa J, Riolobos AS. Early growth hormone $(\mathrm{GH})$ treatment promotes relevant motor functional improvement after severe frontal cortex lesion in adult rats. Behav Brain Res 2013; 247 : 48-58.

5. Lozano R, Naghavi M, Foreman K, Lim S, Shibuya K, Aboyans V, et al. Global and regional mortality from 235 causes of death for 20 age groups in 1990 and 2010: a systematic analysis for the Global Burden of Disease Study 2010. Lancet 2012;380:2095-128.

6. Norton R, Kobusingye O. Injuries. N Engl J Med 2013;368:1723-30.

7. Tatro JB. Melanocortins defend their territory: multifaceted neuroprotection in cerebral ischemia. Endocrinology 2006; 147:1122-5.

8. Mangas A, Yajeya J, González N, Ruiz I, Duleu S, Geffard M, et al. Overexpression of kynurenic acid in stroke: an endogenous neuroprotector? Ann Anat 2017;211:33-8.

9. Mangas A, Yajeya J, González N, Ruiz I, Pernía M, Geffard M, et al. Gemst: a taylor-made combination that reverts neuroanatomical changes in stroke. Eur J Histochem 2017;61:2790.

10. Mangas A, Yajeya J, González N, Ruiz I, Geffard M, Coveñas R. 3-hydroxyanthranilic acid is early expressed in stroke. Eur J Histochem 2016;60:2709.

11. Frati A, Cerretani D, Fiaschi AI, Frati P, Gatto V, La Russa R, et al. Diffuse axonal injury and oxidative stress: a comprehensive review. Int J Mol Sci 2017;18. pii:E2600.

12. Guillemin GJ, Kerr SJ, Smythe GA, Smith DG, Kapoor V, Armati P et al. Kynurenine pathway metabolism in human astrocytes: a paradox for neuronal protection. J Neurochem 2001; 78:842-53

13. López YP, Kenis G, Rutten BP, Myint AM, Steinbusch HW, van den Hove DL. Quinolinic acid-immunoreactivity in the naïve mouse brain. J Chem Neuroanat 2016;71:6-12.

14. Lugo-Huitrón R, Blanco-Ayala $\mathrm{T}$, Ugalde-Muñiz P, Carrillo-Mora P, Pedraza-Chaverrí J, Silva-Adaya D, et al. On the antioxidant properties of kynurenic acid: free radical scavenging activity and inhibition of oxidative stress. Neurotoxicol Teratol 2011; 33:538-47.

15. Gellért L, Fuzik J, Göblös A, Sárközi K, Marosi M, Kis Z, et al. Neuroprotection with a new kynurenic acid analog in the four-vessel occlusion model of ischemia. Eur J Pharmacol 2011;667: 182-7.

16. Pérez-De La Cruz V, Königsberg M, Santamaría A. Kynurenine pathway and disease: an overview. CNS Neurol Disord Drug Targets 2007;6:398-410.

17. Braidy N, Grant R, Brew BJ, Adams S, Jayasena T, Guillemin GJ. Effects of kynurenine pathway metabolites on intracellular NAD+ synthesis and cell death in human primary astrocytes and neurons. Int J Tryptophan Res 2009; 2:61-9.

18. Bohár Z, Toldi J, Fülöp F, Vécsei L. Changing the face of kynurenines and neurotoxicity: therapeutic considerations. Int J Mol Sci 2015;16:9772-93.

19. Quagliariello E, Papa S, Saccone C, Alifano A. Effect of 3-hydroxyanthranilic acid on the mitochondrial res- piratory system. Biochem J 1964;91: 137-46

20. Dykens JA, Sullivan SG, Stern A. Oxidative reactivity of the tryptophan metabolites 3-hydroxyanthranilate, cinnabarinate, quinolinate and picolinate. Biochem Pharmacol 1987;36:211-7.

21. Goldstein LE, Leopold MC, Huang X, Atwood CS, Saunders AJ, Hartshorn M, et al. 3-Hydroxykynurenine and 3hydroxyanthranilic acid generate hydrogen peroxide and promote alpha-crystalline cross-linking by metal ion reduction. Biochemistry 2000;39:7266-75.

22. Morita T, Saito K, Takemura M, Maekawa N, Fujigaki S, Fujii H, et al. 3-hydroxyanthranilic acid, an L-tryptophanmetabolite, induces apoptosis in monocyte-derived cells stimulated by interferon-gamma. Ann Clin Biochem 2001;38:242-51.

23. Kim SY, Senatorov VVJr, Morrissey CS, Lippmann K, Vazquez O, Milikovsky DZ, et al. TGF $\beta$ signaling is associated with changes in inflammatory gene expression and perineuronal net degradation around inhibitory neurons following various neurological insults. Sci Rep 2017;7:7711.

24. Marcet P, Santos N, Borlongan CV. When friend turns foe: central and peripheral neuroinflammation in central nervous system injury. Neuroimmunol Neuroinflamm 2017;4:82-92.

25. O'Farrell K, Fagan E, Connor TJ, Harkin A. Inhibition of the kynurenine pathway protects against reactive microglial-associated reductions in the complexity of primary cortical neurons. Eur J Pharmacol 2017;810:163-73.

26. Agus A, Planchais J, Sokol H. Gut microbiota regulation of tryptophan metabolism in health and disease. Cell Host Microbe 2018;23:716-24.

27. Garrison AM, Parrott JM, Tuñón A, Delgado J, Redus L, O'Connor JC. Kynurenine pathway metabolic balance influences microglia activity: Targeting kynurenine monooxygenase to dampen neuroinflammation. Psychoneuroendocrinology 2018;4:1-10.

28. Sas K, Szabó E, Vécsei L. Mitochondria, oxidative stress and the kynurenine system, with a focus on ageing and neuroprotection. Molecules 2018;23. pii: E191.

29. Heredia M, Palomero J, de la Fuente A, Criado JM, Yajeya J, Devesa J, et al. Motor improvement of skilled forelimb use induced by treatment with growth hormone and rehabilitation is dependent on the onset of the treatment after cortical ablation. Neural Plast 2018; 6125901.

30. Coveñas R, Mangas A, Sánchez ML, 
Cadena D, Husson M, Geffard M. Generation of specific antisera directed against D-amino acids: focus on the neuroanatomical distribution of D-glutamate and other D-amino acids. Folia Histochem Cytobiol 2017;55:177-89.

31. Sánchez ML, Mangas A, Medina LE, Aguilar LA, Díaz-Cabiale Z, Narváez $\mathrm{JA}$, et al. Immunohistochemical mapping of neurotensin in the alpaca diencephalon. Folia Histochem Cytobiol 2018;56:49-58.

32. Paxinos G, Watson C. The rat brain in stereotaxic coordinates. Sydney: Academic Press; 1982.

33. Marcos P, Corio M, Dubourg P, Coveñas R, Tramu G. Double immunocytochemistry in pre-embedding electron microscopy for the detection of neurotensin and tyrosine hydroxylase in the guinea pig, using two primary antisera raised in the same species. Brain Res Brain Res Protoc 1997;2:1-8.

34. Li S, Overman JJ, Katsman D, Kozlov SV, Donnelly CJ, Twiss JL, et al. An age-related sprouting transcriptome provides molecular control of axonal sprouting after stroke. Nat Neurosci 2010;13:1496-504.

35. Blizzard CA, King AE, Haas MA, O'Toole DA, Vickers JC, Dickson TC. Axonal shearing in mature cortical neurons induces attempted regeneration and the reestablishment of neurite polarity. Brain Res 2009;1300:24-36.

36. Barresi MJ, Burton S, Dipietrantonio K, Amsterdam A, Hopkins N, Karlstrom RO. Essential genes for astroglial development and axon pathfinding during zebrafish embryogenesis. Dev Dyn 2010;239:2603-18.

37. Jha MK, Kim JH, Song GJ, Lee WH, Lee IK, Lee HW, et al. Functional dissection of astrocyte-secreted proteins: Implications in brain health and diseases. Prog Neurobiol 2018;162:37-69.

38. Lin X, Miao P, Wang J, Yuan F, Guan Y, Tang $\mathrm{Y}$ et al. Surgery-related thrombosis critically affects the brain infarct volume in mice following transient middle cerebral artery occlusion. PLoS One 2013;8:e75561.

39. Moffett JR, Namboodiri MA. Tryptophan and the immune response. Immunol Cell Biol 2003;81:247-65.

40. Yan Y, Zhang GX, Gran B, Fallarino F, $\mathrm{Yu} \mathrm{S}, \mathrm{Li} \mathrm{H}$, et al. IDO upregulates regulatory $\mathrm{T}$ cells via tryptophan catabolite and suppresses encephalitogenic $\mathrm{T}$ cell responses in experimental autoimmune encephalomyelitis. J Immunol 2010; 185:5953-61.

41. Campbell BM, Charych E, Lee AW, Möller T. Kynurenines in CNS disease: regulation by inflammatory cytokines. Front Neurosci 2014;8:1-22.

42. Gold AB, Herrmann N, Swardfager W, Black SE, Aviv RI, Tennen G, et al. The relationship between indoleamine 2,3dioxygenase activity and post-stroke cognitive impairment. J Neuroinflamm 2011;8:17.

43. Lovelace MD, Varney B, Sundaram G, Lennon MJ, Lim CK, Jacobs K, et al. Recent evidence for an expanded role of the kynurenine pathway of tryptophan metabolism in neurological diseases. Neuropharmacology 2017;112:373-88.

44. Guillemin GJ, Smythe G, Takikawa O, Brew BJ. Expression of indoleamine 2,3-dioxygenase and production of quinolinic acid by human microglia, astrocytes, and neurons. Glia 2005; 49:15-23.

45. Guillemin GJ, Cullen KM, Lim CK, Smythe GA, Garner B, Kapoor V, et al. Characterization of the kynurenine pathway in human neurons. J Neurosci 2007;27:12884-92.

46. Chen Y, Stankovic R, Cullen KM, Meininger V, Garner B, Coggan S, et al. The kynurenine pathway and inflammation in amyotrophic lateral sclerosis. Neurotox Res 2010;18:132-42.

47. Pérez-de la Cruz V, Amori L, Sathyasaikumar KV, Wang XD, Notarangelo FM, Wu HQ, et al. Enzymatic transamination of D-kynurenine generates kynurenic acid in rat and human brain. J Neurochem 2012;120: 1026-35.

48. Sundaram G, Brew BJ, Jones SP, Adams S, Lim CK, Guillemin GJ. Quinolinic acid toxicity on oligodendroglial cells: relevance for multiple sclerosis and therapeutic strategies. J Neuroinflamm 2014;11:204.

49. Lim CK, Fernández-Gómez FJ, Braidy N, Estrada C, Costa C, Costa S, et al. Involvement of the kynurenine pathway in the pathogenesis of Parkinson's disease. Prog Neurobiol 2017;155:76-95.

50. Blight AR, Cohen TI, Saito K, Heyes MP. Quinolinic acid accumulation and functional deficits following experimental spinal cord injury. Brain
1995;118:735-52.

51. Smith DH, Okiyama K, Thomas MJ, McIntosh TK. Effects of the excitatory amino acid receptor antagonists kynurenate and indole-2-carboxylic acid on behavioral and neurochemical outcome following experimental brain injury. J Neurosci 1993;13:5383-92.

52. Yan EB, Frugier T, Lim CK, Heng B, Sundaram G, Tan M, et al. Activation of the kynurenine pathway and increased production of the excitotoxin quinolinic acid following traumatic brain injury in humans. J Neuroinflammation 2015; 12:110.

53. Suma T, Koshinaga M, Fukushima M, Kano T, Katayama Y. Effects of in situ administration of excitatory amino acid antagonists on rapid microglial and astroglial reactions in rat hippocampus following traumatic brain injury. Neurol Res 2008;30:420-9.

54. Koola MM, Sklar J, Davis W, Nikiforuk A, Meissen JK, Sawant-Basak A, et al. Kynurenine pathway in schizophrenia: galantamine-memantine combination for cognitive impairments. Schizophr Res 2018;193:459-60.

55. Koola MM. Galantamine-memantine combination for cognitive impairments due to electroconvulsive therapy, traumatic brain injury, and neurologic and psychiatric disorders: kynurenic acid and mismatch negativity target engagement. Prim Care Companion CNC Disord 2018;20. pii: 17nr02235

56. Sun HY, Li Q, Chen XP, Tao LY. Mismatch negativity, social cognition, and functional outcomes in patients after traumatic brain injury. Neural Regen Res 2015;10:618-23.

57. Lavoie S, Murray MM, Deppen P, Knyazeva MG, Berk M, Boulat O, et al. Glutathione precursor, N-acetyl-cysteine, improves mismatch negativity in schizophrenia patients. Neuropsychopharmacology 2008;33:2187-99.

58. Klauser P, Xin L, Fournier M, Griffa A, Cleusix M, Jenni R, et al. N-acetylcysteine add-on treatment leads to an improvement of fornix white matter integrity in early psychosis: a doubleblind randomized placebo-controlled trial. Transl Psychiatry 2018;8:220.

59. Mangas A, Vecino E, Rodríguez FD, Geffard M, Coveñas R. GEMSP exerts a myelin-protecting role in the rat optic nerve. Neurol Res 2013;35:903-11. 